\title{
Almut Küppers, Torben Schmidt Maik Walter (Hg.) (2011), Inszenierungen im Fremdsprachenunterricht
}

\author{
Grundlagen, Formen, Perspektiven. Braunschweig: Schroedel, \\ Diesterweg, Klinkhardt. ISBN: 978-3-425-71214-7
}

\section{Barbara Schmenk}

Gerade rechtzeitig vor Publikation dieser Ausgabe von Scenario ist der von Almut Küppers, Torben Schmidt und Maik Walter herausgegebenene Band $\mathrm{zu}$ "Inszenierungen im Fremdsprachenunterricht" erschienen, eine Sammlung von Aufsätzen, die sich allesamt mit dramapädagogischen Aspekten und unterrichtspraktischen Vorschlägen beschäftigen. Ein Muss für jede/n dramapädagogisch interessierte/n LeserIn - und auch ein Muss für eine Rezension an diesem Ort.

Inszenierungen, so die HerausgeberInnen einleitend, erschöpfen „sich nicht im klassischen Schultheater", sondern bilden einen eigenen Bereich, der sich „mittlerweile zu einem äußerst dynamischen Anwendungsfeld entwickelt hat, der theoretisch fundiert ist, empirisch erforscht wird und von dem Impulse ausgehen, die dieklassischen fremdsprachendidaktischen Diskussionen bereichern" (S. 5). Entsprechend groß ist die Bandbreite der in den Beiträgen behandelten Ansätze, und entsprechend vielfältig sind die Leseeindrücke und Praxisanregungen, die der Band vermittelt.

Insgesamt 16 Beiträge umfasst der Band (inklusive der Einführung durch die HerausgeberInnen), angelehnt an den Untertitel des Bandes sortiert nach drei Kategorien: Grundlagen, Formen und Perspektiven (wobei sich der Rezensentin nicht ganz erschlossen hat, wie sich die Beiträge unter "Formen" von denen unter "Perspektiven" grundsätzlich unterscheiden; in beiden finden sich Berichte und Analysen von Unterrichtsszenarien und -erfahrungen. Aber das ist eine relativ marginale und für diese Rezension folgenlose Beobachtung).

Den Einstieg in den Band und den ersten Teil („Grundlagen“) macht - eine editorisch korrekte Entscheidung - der dramapädagogische Vordenker und Wegbereiter Manfred Schewe mit dem schönen und wegweisenden Titel „Die Welt auch im fremdsprachlichen Unterricht immer wieder neu verzaubern Plädoyer für eine performative Lehr- und Lernkultur". Schewe entwickelt hierin, in Anlehnung an Erika Fischer-Lichtes (theaterwissenschaftliche) Gedanken zur Ästhetik des Performativen, einige Überlegungen zur ästhetischen Erfahrung im und mit dem fremdsprachlichen Lehren und Lernen. Fischer-Lichtes Ausführungen zu Körper, Stimme, Präsenz und Raum greift er auf und 
unterzieht sie einer dramapädagogischen Prüfung mit dem Ziel, didaktische Aspekte ästhetischer Erfahrung auszuloten. Unter der dreifachen Perspektive von Ereignis, Inszenierung und ästhetischer Erfahrung diskutiert Schewe schließlich die Möglichkeiten, die eine Ästhetik des Performativen auch im Fremdsprachunterricht implizieren könnte. Der Beitrag bereitet somit eine Bühne für die folgenden Beiträge des Bandes, denn Schewes „Verzauberung“ des Klassenraums zum performativen mehrsprachigen und mehrkulturellen Spielraum, in dem ästhetische Erfahrungen gemacht werden, bildet gleichsam ein Tor zu diesem Inszenierungsband.

Es folgt ein Beitrag von Almut Küppers und Andreas Bonnet zum Vergleich von kooperativem Lernen und Dramapädagogik als zwei Formen populärer Inszenierungsformen im Sprachunterricht. Ausgehend vom Begriff der Performativität und seinen spezifischen Bedeutungsdimensionen im Rahmen fremdsprachlichen Lehrens und Lernens vergleichen Bonnet und Küppers Formen des kooperativen Lernens (sowohl Mikromethoden wie Kleingruppenarbeit als auch Makromethoden wie simulierte Interaktionsformen, z. B. Parlamentsdebatten) mit dramapädagogischen Ansätzen, und zwar hinsichtlich der jeweiligen Rolle und den Formen von Performativität. Dieser Ansatz mag in der knappen Skizzierung trocken klingen; der Beitrag von Bonnet und Küppers ist jedoch sowohl gut lesbar als auch konzeptuell sehr aufschlussreich, weil das erarbeitete Raster von Performativität sich als extrem fruchtbar erweist, wenn es um die Analyse und Bewertung von dramapädagogischen und kooperativen Lehr- und Lernformen im FSU geht. Nicht zuletzt ermöglicht die konsequente Einbeziehung der Dimension des Reflektierens v on I nszenierungen ( sei es von Interaktionsrollen, Handlungen, Körperlichkeit im Kontext inszenierter und realweltlicher Interaktionen) eine sehr differenzierte Darstellung und Auffächerung dessen, was genau geschieht und reflektiert wird und werden kann, wenn man Inszenierungen (im engeren und im weiteren Sinne) in den Fremdsprachenunterricht integriert. Der Vergleich von kooperativem Lernen und Dramapädagogik zeigt zudem, wie viel mehr Lern- und Reflexionspotenzial durch dramapädagogische Unterrichtsansätze freigesetzt werden kann, und liest sich so als ein Plädoyer für die Ästhetisierung von unterrichtlicher Kooperation und Interaktion. Lernende erfahren mehr und werden zu weit mehr Nachdenken, Kreativität und sozialer Interaktion - also zur Entwicklung von performativer Kompetenz - angeregt, wenn sie nicht nur mit ihren peers im Klassenraum (sprachlich und projektbezogen) kooperieren, sondern wenn sie dies in eigens gestalteten Spielräumen tun, in denen sie durch die Übernahme neuer Rollen und Aufgaben ihre performative Kompetenz erweitern und differenzieren können. Die damit mögliche Doppelperspektive (Ich als ein anderer und in einer anderen Sprache) stellt eine wichtige Voraussetzung dafür dar, nicht nur die Fiktivität fremdsprachenunterrichtlicher Interaktion und Rollenzuweisungen kreativ zu unterminieren, sondern auch die eigene Person jeweils anders $\mathrm{zu}$ inszenieren - und zugleich über die dabei erfahrbare und erspielbare Mehrperspektivität reflektieren z u können. K üppers u nd Bonnet liefern mit diesem Beitrag somit ein wichtiges - und bislang nicht verfügbares 
- Scharnier für die Verbindung von Theorie und Praxis dramapädagogischen Arbeitens im Fremdsprachenunterricht.

Der dritte Beitrag unter der Rubrik „Grundlagen“ stellt einen Übergang zur Unterrichtspraxis dar und greift die wichtige Frage der Lehrerbildung auf. Adrian Haack und Carola Surkamp berichten von Lehramtsseminaren an der Universität Göttingen, in denen angehende Lehrende nicht nur dramapädagogische Arbeitsformen am eigenen Leibe kennenlernen, sondern auch in Kooperation mit Göttinger Schulen selber Unterrichtssequenzen und Theaterprojekte erarbeiten und ausprobieren. Im Ergebnis führt diese Form der Kooperation somit zu einer Verschränkung performativer Erfahrungen und Aktivitäten: SchülerInnen sind ebenso Teil von Inszenierungen wie angehende Lehrende, und sowohl schulisches wie auch universitäres Lehren und Lernen wird zunehmend verschränkt und verschwimmt schließlich vor dem Horizont dramapädagogischer Aktions- und Reflexionsformen. H aack u nd Surkamp liefern mit diesem Beispiel somit einen wichtigen Baustein für die Integration von Dramapädagogik sowohl in den unterrichtlichen Alltag als auch in die universitäre Lehrerbildung.

Die übrigen Beiträge des Bandes behandeln jeweils konkrete Unterrichtsprojekte. Den Einstieg in den Teilbereich „Formen“ bildet der Beitrag von Susanne Even, die eine Sequenz aus dem DaF-Unterricht zur Behandlung der Wechselpräpositionen schildert. Durch den Einsatz von Ungeziefer gelingt ihr eine besonders nachhaltige Inszenierung dieses bei Lernenden sonst eher ungeliebten grammatischen Phänomens: Ein fliegender Käfer (sicher auch durch andere Insekten oder Flugobjekte ersetzbar) dient zur Inszenierung von Richtungen und Orten im Klassenraum, deren Versprachlichung entsprechend zur Klärung und Verdeutlichung der Kasuswahl nach Wechselpräpositionen führt. Even erläutert dies nicht nur hinsichtlich der dramapädagogischen Idee, sondern geht genau auf jeden einzelnen Unterrichtsschritt ein - der Beitrag liefert insofern ein Grundgerüst für dramapädagogisches Arbeiten mit sprachlichen Strukturen (selbstverständlich auch teilweise übertragbar auf andere sprachliche und kulturelle Phänomene).

Michael Legutke und Steffi Vogt widmen sich in ihrem Beitrag der mittlerweile in Englischlehrwerken vor allem für die Primarstufe obligatorischen Handpuppe. Da Schulbuchverlage in der Regel solche Handpuppen für den Unterricht zum Kauf anbieten, den Einsatz jedoch kaum genauer erläutern oder didaktisch reflektieren, besteht hier echter Bedarf in Sachen Erläuterung, Begründung und Nutzen des Handpuppeneinsatzes. Dass dies in einem Band zur Dramapädagogik geschieht, ist folgerichtig und eröffnet eine Reihe von Möglichkeiten und Perspektiven für den Spielraum Fremdsprachenunterricht, denn die Handpuppe kann und soll im Unterricht weit mehr sein als einfach nur eine kindgerechte Figur, die Übungen mit Kindern begleitet.

Die Idee einer Sprachstadt, in der sich verschiedene Orte bzw. Institutionen (wie Geschäfte, Ämter etc.) befinden, d ie v on S chülerInnen b esucht werden müssen, schildert Carmen Bietz in ihrem Aufsatz. Hierbei geht es um ein relativ aufwändiges Projekt, an dem auch die TeilnehmerInnen eines 
Didaktikseminars beteiligt waren, die die verschiedenen Sprachstadt-Orte (oder genauer: Stationen) gestalteten und Aufgaben dafür entwickelten, wobei sie auch zum großen Teil selbst aktiv an einzelnen Stationen mitarbeiteten (Telefonate führen, Gespräche führen). Die SchülerInnen erhalten für den Gang durch die Sprachstadt einen „Laufzettel“ mit (Interaktions-)Aufgaben, die am jeweiligen Lernort erledigt werden müssen. An der Grenze zwischen Fiktion und Realität gelingt es mit diesen Sprachstadt-Stationen so, SchülerInnen zu möglichst authentischem Sprachgebrauch zu motivieren, ohne dass sie ganz dem „Ernstfall“ ausgesetzt sind. Die LehramtskandidatInnen, die an den Stationen arbeiteten, dürften an diesem Projekt ebenso gelernt haben wie die SchülerInnen - also ein echtes Lehr-Lern-win-win-Szenario.

Es folgen vier Beiträge, die sich ausführlich mit Inszenierungen von Texten auseinandersetzen. Alle geben wertvolle Tipps und Hinweise für die Theaterarbeit und dürften vor allem denjenigen von Nutzen sein, die selbst eine Theaterproduktion durchführen wollen. Susanne Franz und Mechthild Hesse skizzieren die Theaterproduktionen, die sie an der PH Freiburg mit Studierenden durchgeführt haben, wobei die Produktionen jeweils auch für Schulgruppen aufgeführt wurden. Damit einher geht auch die didaktische Begleitung und Fortbildung für Englischlehrende, die nicht nur die literarische Vorlage genau kennenlernen, sondern diese auch im Unterricht behandeln und erspielen können, so dass der Besuch der studentischen Theateraufführung dann eine Art Höhepunkt auch der unterrichtlichen Textarbeit darstellt. Maik Walter berichtet von einem Projekt mit ausländischen Studierenden, mit denen er eine Erzähltheaterproduktion in Berlin durchgeführt hat. Hier geht es nicht nur um die Produktion eines spezifischen S tückes (Judith H ermanns „Sommerhaus, später"), sondern der Beitrag gibt eine Einführung in das Erzähltheater und Formen des Erzählens im dramapädagogischen Unterricht. Wolfgang Poreda schildert die Theaterarbeit mit SchülerInnen der Oberstufe, die im Rahmen einer Arbeitsgruppe ein Stück selbst schrieben und in Szene setzten. Großprojekte dieser Art sind bekanntermaßen sehr schwierig; umso wichtiger ist es, solche Projekte bekannt zu machen und andere zu ermutigen, sich nicht von der vielen Arbeit abschrecken zu lassen und es einfach zu probieren. Ebenso wie die SchülerInnen brauchen Lehrkräfte für solche Aufgaben schließlich nicht nur das nötige Know-how und Interesse, sondern auch eine gehörige Portion Idealismus und Risikobereitschaft. Ingrid Stritzelberger geht in ihrem Beitrag schließlich auf eine multimediale Inszenierung ein, in der nicht nur traditionell Theater gespielt, sondern auch andere Inszenierungsformen zum Tragen kommen (Powerpoint-Präsentationen, Gedichtrezitationen, Fotoshows, etc.). Im Ergebnis ist diese Produktion dann ein echtes Multimediaspektakel und zeigt, wie viel Zusatz-Potenzial auch in medialen Umgebungen liegt, wenn man einmal anfängt, dramapädagogisch zu arbeiten.

Im dritten Teil des Bandes („Perspektiven“) finden sich zunächst zwei Beiträge, die Forschungsprojekte zur Wirkung und Wirksamkeit dramapädagogischen Arbeitens beschreiben. Heike Wedel und Anja Jäger widmen sich jeweils spezifischen dramapädagogischen Unterrichtssequenzen und gehen insbesondere auf 
die Bedeutung dieser für die Entwicklung von interkultureller kommunikativer Kompetenz (IKK, Byram 1997) ein. Das ist insofern bemerkenswert, als es zeigt, dass es gerade der Bereich des Interkulturellen zu sein scheint, in dem Forschende die größte Wirkung dramapädagogischen Arbeitens vermuten. Der Grund dafür ist wohl, dass mit Interkulturalität immer Aspekte wie Perspektivwechsel, Empathie, Verstehen des Anderen und damit Rollendistanz und Reflexion verbunden sind. Ganz offenbar lassen sich dramapädagogische Ansätze nicht darauf reduzieren, Sprachkompetenzen zu fördern, sondern sie betreffen Personen sehr viel direkter und persönlicher, womit man im Bereich der übergeordneten Bildungs- und Erziehungsziele von Unterricht ist. Hier ist es bekanntermaßen schwierig, Lernprozesse oder gar Bildungsprozesse empirisch zu beobachten. Dass es gerade Byrams Modell von IKK ist, das in den Arbeiten als theoretische Folie genutzt wird, ist wahrscheinlich dadurch zu erklären, dass sein Versuch, IKK aufzusplitten und in handhabbare (und operationalisierbare) Einzelkompetenzen zu zerlegen, ein Gerüst für Forschende bietet, die ihre Ergebnisse ordnen und hinsichtlich eines Modells auswerten wollen. Als LeserIn ist man deshalb direkt eingeladen, auch über alternative Modelle und Ansätze nachzudenken, die das Lernpotenzial von Drama im FSU erfassen könnten. Solche Überlegungen erinnern uns auch daran, dass man als Forschende/ $r$ oft in der Gefahr bzw. unter dem Druck steht, dramapädagogische Ansätze bzw. ihre Ergebnisse messbar und quantifizierbar machen zu müssen. Damit allerdings kann man vieles von dem, was man als dramapädagogisch arbeitende/r Lehrende/r erlebt, nicht adäquat begreifen. Denn zweifellos löst Drama im Unterricht zahlreiche Spiel-, Denk- und Lernprozesse aus - viele davon überraschend und unvorhersehbar. Wenn man diese in einer Forschungsstudie erfassen will, indem man ein Raster von vorformulierten und fein gestaffelten Kompetenzen anlegt, läuft man Gefahr, vieles zu übersehen, das von diesen Rastern nicht erfasst wird - und auch nicht erfasst werden kann.

Jenny Passon schildert eine AG an einer Realschule, in der Shakespeare inszeniert wurde. Der Beitrag ist ermutigend gleich in mehrfacher Hinsicht: Denn erstens ist Shakespeare durchaus auch für Nicht-GymnasiastInnen geeignet (Passon hatte eine Textvorlage mit dem schönen Titel Othello@realschule.de erstellt, die als modernisierte Othello-Version sowohl Originalpassagen als auch modernisierte Teile enthält), zweitens haben sie sehr viel Spaß und fühlen sich stolz, wenn sie erfolgreich den sicherlich einschüchternden „größten englischsprachigen Autor" bezwingen. Drittens machen Passons Ausführungen auch deutlich, wieviel von Shakespeares Sprache die SchülerInnen gelernt haben und schätzen. Es scheint gerade das Vorurteil zu sein, dass RealschülerInnen nicht Shakespeare lesen können, das sie besonders motiviert hat, das Gegenteil zu beweisen.

Den Abschluss des dritten Teils des Bandes bilden schließlich zwei Beiträge von Torben Schmidt und Katrin Biebrighäuser, die die Inszenierungen in den virtuellen Raum hineinverlegen. Schmidt schildert eine Unterrichtseinheit (genauer: eine Projektarbeitssequenz) zur Inszenierung von Ann Chisletts Flippin' In in der Klasse 11, wobei die SchülerInnen die Textvorlage bearbeiten, 
also selbst Szenen schreiben und proben mussten, um schließlich Videos von verschiedenen Szenen zu erstellen, die fast alle auf YouTube veröffentlicht und von einer anderen Klasse an einer Schule im Nachbarort angeschaut und kommentiert wurden. Biebighäuser beschreibt eine Unterrichtssequenz, bei der die Inszenierung selbst bereits im virtuellen Raum stattfindet, in Second Life. SchülerInnen gestalten selbst je eigene Avatare und begegnen einander - über zahlreiche Grenzen hinweg, denn virtuelle Plattformen machen es möglich, dass nicht nur sprachliche und kulturelle, sondern auch räumliche und zeitliche Dimensionen einfach übersprungen werden können. Konkret schildert sie ein Projekt, bei dem Lernende (Studierende aus Gießen und aus Breslau) auf Second Life in die DDR der 60er Jahre versetzt werden (Berlin-Marzahn 1967) und sich über ihre Erfahrungen austauschen. Schon diese Kurzbeschreibung macht deutlich, dass es in dergleichen Szenarios ungleich mehr Möglichkeiten der Inszenierung und der Begegnung gibt - dass allerdings mit der zunehmenden Virtualisierung auch die eigene Körperlichkeit - in der Performativität und Theaterästhetik zweifellos zentral - gleich mit virtualisiert wird.

Insgesamt zeigt der Band eine bemerkenswerte Vielfalt von Ideen und gehört in das Bücherregal all derjenigen, die sich für Dramapädagogik interessieren seien das Lehrende, LehrerInnenaus- und -fortbildende oder Forschende. Und es ist zu hoffen, dass er auch möglichst vielen in die Hände gerät, die bislang noch eher zögerlich oder gar eher abwehrend gegenüber dramapädagogischen Ansätzen im Fremdsprachenunterricht sind. Man kann den Band als Fundgrube und auch als aktuellen Spiegel dramapädagogischer Theorie und Praxis in Deutschland begreifen, und deshalb ist er mehr als willkommen und sei allen Scenario-LeserInnen an dieser Stelle wärmstens empfohlen. 